\title{
Surface Tension of Molten Glasses in the System Barium Oxide-Boric Oxide-Silica
}

\author{
Herman F. Shermer
}

\begin{abstract}
The surface tension of 154 compositions in the system $\mathrm{BaO}-\mathrm{B}_{2} \mathrm{O}_{3}-\mathrm{SiO}_{2}$ were measured by a maximum pull-on-cylinder method, over the approximate range $900^{\circ}$ to $1,300^{\circ} \mathrm{C}$. The region of glass formation was investigated along with the adjacent regions. Several compositions were studied in the two-immiscible-liquid region. In this two-liquid region the lines of constant surface tension followed the tie lines in the phase diagram. Outside the two-liquid region the surface tension was increased by the addition of $\mathrm{BaO}$ and to a lesser degree by $\mathrm{SiO}_{2}$. At $900^{\circ} \mathrm{C}$ the surface tension was relatively high near the composition corresponding to the $3 \mathrm{BaO} \cdot 3 \mathrm{~B}_{2} \mathrm{O}_{3} \cdot 2 \mathrm{SiO}_{2}$ compound, which has a melting point of $1,009^{\circ} \mathrm{C}$.
\end{abstract}

\section{Introduction}

A study of the surface tension of the system $\mathrm{BaO}-\mathrm{B}_{2} \mathrm{O}_{3}-\mathrm{SiO}_{2}$ was undertaken as part of a comprehensive investigation of various physical properties of simple glass-forming systems [1 to 13]. ${ }^{1}$ The surface tension of the binary system, $\mathrm{BaO}-\mathrm{B}_{2} \mathrm{O}_{3}$, has been reported as part of a previous investigation of the properties of the binary alkaline-earth borates [10]. The range of compositions studied was limited by crystallization and by relatively high viscosity in the region of high silica. The phase diagrams for the systems $\mathrm{BaO}-\mathrm{B}_{2} \mathrm{O}_{3}$ and $\mathrm{BaO}-\mathrm{B}_{2} \mathrm{O}_{3}$ $\mathrm{SiO}_{2}$ show a two-liquid region at low concentrations of alkaline-earth oxide.

The system $\mathrm{BaO}-\mathrm{B}_{2} \mathrm{O}_{3}-\mathrm{SiO}_{2}$ is of fundamental importance in the ceramic field and especially in the glass industry, as it serves as a starting point for investigations of a large class of barium-crown glasses.

\section{Experimental Procedure}

\subsection{Preparation of Glasses}

The composition of the glasses used in this investigation is shown in table 1 . The glasses were made by melting at $1,300^{\circ}$ to $1,500^{\circ} \mathrm{C}$ the required amounts of chemicals in platinum crucibles and stirring the melts with platinum stirrers until they appeared homogeneous. The melts varied in weight from 100 to $500 \mathrm{~g}$.

Chemical-analysis samples were taken from the melts before and after surface-tension measurement. The reported compositions are the average of the two determinations unless otherwise noted in table 1. The $\mathrm{BaO}$ and $\mathrm{SiO}_{2}$ contents were determined gravimetrically and the $\mathrm{B}_{2} \mathrm{O}_{3}$ by difference. Small changes were noted between the batch composition and the first analysis. A further loss of a few tenths of a percent of $\mathrm{B}_{2} \mathrm{O}_{3}$ was noted between the two

1 Figures in brackets indicate the literature references at the end of this paper. analyses, indicating volatilization during the tests. The estimated probable errors of the gravimetric determinations are about \pm 0.1 weight percent. ${ }^{2}$

\subsection{Method of Test}

The method of measuring surface tension used in this investigation has been previously described in detail [14]. It consists in determining the pull exerted by the test surface on a thin-walled platinum cylinder during the removal of its lower edge from contact with the liquid. The first determination of the surface tension of any melt was made at $1,300^{\circ} \mathrm{C}$. Subsequent measurements were made at 100-deg C intervals, decreasing the temperature until the glass was too viscous to yield in a reasonable time or until crystallization occurred.

The density values used in the correction of the surface-tension calculations were determined by interpolation between room-temperature values for each composition and a few known values in the temperature and cómposition range of interest. For convenience in presenting the data, all values of surface tension have been adjusted to even 100-deg intervals. The temperature of any measurement may have been $4 \mathrm{deg} \mathrm{C}$ from that given in table 1 .

\section{Results and Discussion}

Surface-tension data are given in table 1. Differences in batch materials and source of glasses are indicated in the footnotes. Figure 1 shows the isoepitatic [15], or constant surface-tension, lines at $1,300^{\circ} \mathrm{C}$ and the locations of the compositions investigated. The surface tension increased with decrease in $\mathrm{B}_{2} \mathrm{O}_{3}$ content. This figure also contains an outline of the two-immiscible-liquid region and the conodes, i. e., the tie lines connecting the two conjugate compositions in the phase diagram as determined by Levin and Ugrinic [16].

In the two-liquid region the isoepitatic lines approximate the conodes. This is in accord with the

2 The samples were analyzed by Ralph I. Cohen, Ernest B. Clark, and J. Richard Spann of the Bureau. 
TABLE 1. Surface tension of composition in the system $\mathrm{BaO}-\mathrm{B}_{2} \mathrm{O}_{3}-\mathrm{SiO}_{2}$

\begin{tabular}{|c|c|c|c|c|c|c|c|}
\hline \multicolumn{3}{|c|}{ Compositionª } & \multicolumn{5}{|c|}{ Surface tension ${ }^{b}$} \\
\hline $\mathrm{BaO}$ & $\mathrm{B}_{2} \mathrm{O}_{3}$ & $\mathrm{SiO}_{2}$ & $1,300^{\circ} \mathrm{C}$ & $1,200^{\circ} \mathrm{C}$ & $1,100^{\circ} \mathrm{C}$ & $1,000^{\circ} \mathrm{C}$ & $900^{\circ} \mathrm{C}$ \\
\hline$w t \%$ & $w t \%$ & $w t \%$ & $d / \mathrm{cm}$ & $d / \mathrm{cm}$ & $d / \mathrm{cm}$ & $\mathrm{d} / \mathrm{cm}$ & $d / \mathrm{cm}$ \\
\hline $\begin{array}{l}-0.0 \\
\circ 4.3\end{array}$ & $\begin{array}{r}100.0 \\
95.7\end{array}$ & - & 96.3 & 93.4 & $\begin{array}{l}88.2 \\
88.6\end{array}$ & $\begin{array}{l}83.5 \\
84.0\end{array}$ & $\begin{array}{l}80.5 \\
80.0\end{array}$ \\
\hline - 4.9 & 95.1 & - n... & 94.8 & 90.9 & 86.3 & 82.8 & \\
\hline $\begin{array}{l}\text { c15. } 5 \\
\text { ○24.9 }\end{array}$ & $\begin{array}{l}84.5 \\
75.1\end{array}$ & $-\cdots$ & $\begin{array}{r}96.3 \\
106.3\end{array}$ & $\begin{array}{l}91.8 \\
99.8\end{array}$ & 87. 2 & $\begin{array}{l}82.4 \\
89.9\end{array}$ & 79.4 \\
\hline 30.1 & 69.9 & $-\cdots$ & 121.6 & 1198 & 116.4 & 114.7 & 115.3 \\
\hline е32. 3 & 67.7 & - & 122.2 & 120.0 & 117.8 & 116.2 & 100 \\
\hline 35.0 & 65.0 & -...- & 134. 3 & 133. 4 & 133. 3 & 136. 9 & 140.7 \\
\hline c35. 8 & 64. 2 & -...- & 135.4 & 137.6 & 137. 5 & 141. 0 & 145.7 \\
\hline 36.6 & 63.4 & $\cdots--$ & 139.7 & 140.7 & 142.1 & 145.8 & 150.5 \\
\hline e40. 8 & 59. 2 & -.. & 156.0 & 157.9 & 161.1 & 165.8 & 171.4 \\
\hline 41.8 & 58.2 & -.... & 163.8 & 166.6 & 170.4 & 174.7 & \\
\hline 41. 9 & 58. 1 & -...- & 163.5 & 168. 9 & 172. 4 & 177. 0 & \\
\hline 045.3 & 54.7 & $-\cdots$ & 180.6 & 183. 3 & 187. 3 & 192.3 & 197. 2 \\
\hline 47.2 & 52.8 & & 190.0 & 192.2 & 197.0 & 202.4 & 211.0 \\
\hline c47. 6 & 52. 4 & -...- & 190.7 & 193. 7 & 198.0 & 202.9 & 207.3 \\
\hline c51. 0 & 49. 0 & -.-- & 203.5 & 207.4 & 211. 2 & 216. 1 & 220.6 \\
\hline 51.4 & 48.6 & $-\cdots-$ & 206.5 & 210.1 & 213.8 & 218. 9 & \\
\hline $\begin{array}{l}51.5 \\
52.4\end{array}$ & $\begin{array}{l}48.5 \\
47\end{array}$ & $-\cdots$ & $\begin{array}{l}207.3 \\
020\end{array}$ & 211.0 & $\begin{array}{l}214.6 \\
216.8\end{array}$ & 219. 3 & 224.1 \\
\hline 02.4 & 47.6 & --.- & 208.9 & 212.1 & 216.8 & 221.3 & -.... \\
\hline $\begin{array}{r}53.6 \\
055.6\end{array}$ & $\begin{array}{l}46.4 \\
44.4\end{array}$ & $\cdots-$ & $\begin{array}{l}212.5 \\
220.7\end{array}$ & 216. 6 & $\begin{array}{l}221 . \\
228.9\end{array}$ & $\begin{array}{l}226.9 \\
233.7\end{array}$ & 2390 \\
\hline $\begin{array}{l}50.6 \\
56.1\end{array}$ & $\begin{array}{l}44.4 \\
43.9\end{array}$ & ..... & 219.6 & $\begin{array}{l}224.8 \\
224.5\end{array}$ & $\begin{array}{l}228.9 \\
229.4\end{array}$ & $\begin{array}{l}233.7 \\
235.4\end{array}$ & 239.0 \\
\hline 56.4 & 43.6 & $-\cdots$ & 221.1 & 227.0 & 232.0 & 238.3 & $\ldots$ \\
\hline 60.0 & 40.0 & $-\cdots$ & 234.0 & 239.1 & 244.4 & 251.2 & -...- \\
\hline 60.2 & 39.8 & - & 234.8 & 240.4 & 246.0 & 252.7 & \\
\hline • 60.4 & 39. 6 & -...-. & 231. 9 & 237.3 & 243. 2 & 248.4 & - - \\
\hline '65. 1 & $\begin{array}{l}34.9 \\
20.9\end{array}$ & - - & 242. 9 & 251.1 & 257.4 & 263.4 & - - \\
\hline 70. 1 & $\begin{array}{r}29.9 \\
\end{array}$ & -... & 252.4 & 261.7 & 269.0 & 275.1 & -..- \\
\hline $\begin{array}{l}\text { c } 74.2 \\
\text { c79.5 }\end{array}$ & $\begin{array}{l}25.8 \\
20.5\end{array}$ & $-\cdots$ & $\begin{array}{l}263.4 \\
293.9\end{array}$ & $\begin{array}{l}274.0 \\
305.2\end{array}$ & $\begin{array}{l}282.4 \\
314.6\end{array}$ & $\begin{array}{l}288.9 \\
321.6\end{array}$ & $-\cdots$ \\
\hline 2. 1 & 92.9 & 5.0 & 99.3 & 93.3 & & 87.1 & 84.9 \\
\hline $\begin{array}{l}4.9 \\
\end{array}$ & 84.1 & 11. 0 & 107.1 & 103.3 & 97. 9 & 92.8 & 93.9 \\
\hline $\mathrm{d} 10.4$ & 77. 2 & 12. 4 & 113. 8 & 108. 2 & 103. 8 & 100.5 & 97.0 \\
\hline $\mathrm{d} 10.4$ & 65.0 & 24. 6 & 133.5 & 128.7 & 121. 5 & 117. 9 & 116.0 \\
\hline $\mathrm{d} 10.6$ & 52. 9 & 36.5 & 152.5 & 148.6 & 145.7 & 143.8 & $\cdots$ \\
\hline d22. 8 & 65.0 & 12. 2 & 133. 4 & 126.7 & 118.8 & 114.8 & 114. 0 \\
\hline $\begin{array}{l}\mathrm{d} 23.5 \\
\mathrm{~d} 22.0\end{array}$ & $\begin{array}{l}52.9 \\
41.4\end{array}$ & $\begin{array}{l}23.6 \\
36.6\end{array}$ & 146. 5 & 140.4 & 141.0 & 140.2 & 137.3 \\
\hline 29.8 & 67.2 & $\begin{array}{r}0.0 \\
3.0\end{array}$ & $\begin{array}{l}14.1 \\
120.7\end{array}$ & $\begin{array}{l}17.2 \\
119.6\end{array}$ & 113.8 & 110.9 & 110.1 \\
\hline 29.6 & 61. 2 & 9.2 & 132.8 & 128.9 & 123.5 & 118.2 & 124.9 \\
\hline 30.7 & 57.7 & 11. 6 & 142. 0 & 138. 9 & 135. 7 & 132.6 & 135.4 \\
\hline $\begin{array}{l}31.2 \\
33.8\end{array}$ & $\begin{array}{l}63.2 \\
20.4\end{array}$ & $\begin{array}{r}5.6 \\
45.8\end{array}$ & $\begin{array}{l}\text { 132. } 4 \\
241.9\end{array}$ & 128.9 & 126.5 & 125.5 & 124.9 \\
\hline $\mathrm{d} 35.5$ & 40. 2 & 24.3 & 185.2 & 182. 9 & 178.1 & 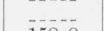 & (c) \\
\hline 36.9 & 57.4 & 5.7 & 153.2 & 155.2 & 155.2 & 158.8 & 167.2 \\
\hline 37. 0 & 45. 9 & 17.1 & 178.7 & 179.8 & 181.3 & 182.6 & 187. 0 \\
\hline 37.3 & 51. 6 & 11.1 & 164. 0 & $\begin{array}{l}165.6 \\
\end{array}$ & 167. 0 & 170. 2 & 179.8 \\
\hline 37.4 & 39. 2 & 23. 4 & 193.4 & 194. 2 & 193. 5 & 193.7 & -.-- \\
\hline 42.1 & 55.3 & 2. 6 & 172.4 & 175. 6 & 178.7 & 184. 0 & 1070 \\
\hline 42.2 & 52.5 & 5.3 & 178.5 & 181.7 & 185.9 & 191.1 & 197. 6 \\
\hline 42. 3 & 49. 6 & 8.1 & 182.5 & 186. 2 & 187. 5 & 193. 8 & 200.9 \\
\hline 42. 3 & 46. 8 & 10. 9 & 188.8 & 193. 7 & 197. 7 & 202.5 & 212. 0 \\
\hline 42.7 & 43.6 & 13. 7 & 196.1 & 199.6 & 202.7 & 207.7 & 216.4 \\
\hline 42.6 & 43.6 & 13.8 & 196.6 & 199. 0 & 202. 2 & 206.1 & 216.8 \\
\hline 42.7 & 40.7 & 16.6 & 203.6 & 206.6 & 209.7 & 214.3 & 221.3 \\
\hline 42.8 & 37.8 & 19.4 & 211.6 & 212.5 & 215.5 & 219.8 & 226. 2 \\
\hline 43.0 & $\begin{array}{l}34.3 \\
21\end{array}$ & 22.7 & 219. 2 & 220.5 & 221.8 & 225. 2 & 231. 2 \\
\hline 43.0 & $\begin{array}{l}31.9 \\
98\end{array}$ & 25.1 & 224. 0 & 225.7 & 226. 0 & 229.8 & 235.1 \\
\hline 43. 2 & $\begin{array}{r}28.9 \\
380\end{array}$ & $\begin{array}{l}27.9 \\
\text { 28 }\end{array}$ & 233.7 & 235. 6 & $\begin{array}{l}236 . \\
2\end{array}$ & 241.5 & -... \\
\hline 43.1 & 28.9 & 28.0 & 232.9 & 234.3 & 235.1 & 238.5 & - \\
\hline 43. 2 & 26.0 & 30.8 & 241.4 & 241.6 & 241.1 & & \\
\hline 47.6 & $\begin{array}{l}20.0 \\
\end{array}$ & 5. 2 & 202.7 & & 210. 2 & 214.4 & 221.1 \\
\hline 47.6 & 44.6 & 7.8 & 206. 2 & 211. 0 & 214.8 & 219.6 & 227.6 \\
\hline 47.8 & 41.7 & 10.5 & 213. 2 & 216.9 & 220.6 & 225.9 & 234.5 \\
\hline 48.1 & 36.0 & 15.9 & 227.1 & 229.6 & 232. 2 & 236.9 & 243.6 \\
\hline 48. 5 & 30.5 & 21.0 & 241. 2 & 243.8 & 246. 9 & 251.6 & 260.3 \\
\hline $\begin{array}{l}48.1 \\
48,3\end{array}$ & 30.4 & $\begin{array}{l}21.5 \\
24.5\end{array}$ & $\begin{array}{r}240.4 \\
2458\end{array}$ & 243.1 & $\begin{array}{r}246.6 \\
252\end{array}$ & 252.4 & $\begin{array}{l}260.5 \\
264\end{array}$ \\
\hline $\begin{array}{l}48.3 \\
48\end{array}$ & 27.7 & $\begin{array}{l}24.0 \\
24.2\end{array}$ & 245.8 & $\begin{array}{l}248.7 \\
249.8\end{array}$ & $\begin{array}{l}252.6 \\
252\end{array}$ & $\begin{array}{l}257.5 \\
257.5\end{array}$ & $\begin{array}{l}264.6 \\
2\end{array}$ \\
\hline $\begin{array}{l}48.4 \\
48.5\end{array}$ & $\begin{array}{l}27.4 \\
24.7\end{array}$ & $\begin{array}{l}24.2 \\
26.8\end{array}$ & $\begin{array}{l}246.4 \\
254.1\end{array}$ & $\begin{array}{l}249.8 \\
257.1\end{array}$ & $\begin{array}{l}252.7 \\
255.8\end{array}$ & $\begin{array}{l}257.5 \\
262.8\end{array}$ & $\begin{array}{l}264.2 \\
272.8\end{array}$ \\
\hline 48.5 & 24.7 & 20.8 & 204.1 & 257.1 & 255.8 & 202.8 & 27.0 \\
\hline $\begin{array}{l}48.7 \\
48.9\end{array}$ & $\begin{array}{l}21.6 \\
18.7\end{array}$ & $\begin{array}{l}29.7 \\
32.4\end{array}$ & $\begin{array}{l}261.9 \\
270.0\end{array}$ & $\begin{array}{l}264.1 \\
272.0\end{array}$ & $\begin{array}{l}265.3 \\
273.4\end{array}$ & $\begin{array}{l}269.9 \\
277.3\end{array}$ & $\begin{array}{l}274.9 \\
282.6\end{array}$ \\
\hline 49.5 & 12.5 & 38.0 & 291. 9 & 291.8 & 291. 4 & 294.6 & \\
\hline 49. 3 & 9.7 & 41. 0 & 302.1 & 301.9 & 302.0 & . & -.... \\
\hline 49.7 & 6.2 & 44.1 & 318.4 & - & & -.-.- & \\
\hline 51. 4 & 15. 3 & 33.3 & 284.0 & 285.5 & 286. 2 & 290.5 & \\
\hline 51.7 & 46.4 & 1.9 & 212.1 & 215.7 & 219.6 & 225.4 & 229.3 \\
\hline 51.8 & 44. 7 & 3.5 & 215. 9 & 219.4 & 223. 2 & 228. 2 & 233. 6 \\
\hline 50. & 43. 2 & 5. 3 & 219. 6 & 223.3 & 227.0 & 231.4 & 238.7 \\
\hline 52,0 & 42. 3 & 5.7 & 220.3 & 224.0 & 227.6 & 232.7 & 239.6 \\
\hline
\end{tabular}

\begin{tabular}{|c|c|c|c|c|c|c|c|}
\hline \multicolumn{3}{|c|}{ Composition ${ }^{2}$} & \multicolumn{5}{|c|}{ Surface tensionb } \\
\hline $\mathrm{BaO}$ & $\mathrm{B}_{2} \mathrm{O}_{3}$ & $\mathrm{SiO}_{2}$ & $1,300^{\circ} \mathrm{C}$ & $1,200^{\circ} \mathrm{C}$ & $1,100^{\circ} \mathrm{C}$ & $1,000^{\circ} \mathrm{C}$ & $900^{\circ} \mathrm{C}$ \\
\hline $\begin{array}{r}w t \% \\
52.0 \\
52.2 \\
52.0 \\
52.3 \\
52.2\end{array}$ & $\begin{array}{r}w t \% \\
39.1 \\
37.8 \\
36.9 \\
36.6 \\
36.4\end{array}$ & $\begin{array}{r}w t \% \\
8.9 \\
10.0 \\
11.1 \\
11.1 \\
11.4\end{array}$ & $\begin{array}{l}d / \mathrm{cm} \\
226.6 \\
228.1 \\
230.9 \\
228.8 \\
231.4\end{array}$ & $\begin{array}{l}d / \mathrm{cm} \\
230.7 \\
231.9 \\
234.3 \\
232.1 \\
235.0\end{array}$ & $\begin{array}{c}d / \mathrm{cm} \\
234.3 \\
235.8 \\
238.2 \\
236.5 \\
238.7\end{array}$ & $\begin{array}{c}d / c m \\
239.7 \\
241.2 \\
242.6 \\
240.6 \\
243.3\end{array}$ & $\begin{array}{l}d / c m \\
247.7 \\
248.3 \\
249.5 \\
247.6 \\
246.3\end{array}$ \\
\hline $\begin{array}{l}52.5 \\
52.4 \\
52.7 \\
52.8 \\
53.1\end{array}$ & $\begin{array}{l}35.3 \\
33.7 \\
31.9 \\
30.7 \\
26.5\end{array}$ & $\begin{array}{l}12.2 \\
13.9 \\
15.4 \\
16.5 \\
20.4\end{array}$ & $\begin{array}{l}229.0 \\
225.3 \\
242.5 \\
243.3 \\
255.2\end{array}$ & $\begin{array}{l}231.9 \\
240.7 \\
246.1 \\
248.1 \\
258.7\end{array}$ & $\begin{array}{l}235.8 \\
244.3 \\
250.3 \\
251.7 \\
262.2\end{array}$ & $\begin{array}{l}240.5 \\
249.3 \\
254.6 \\
256.4 \\
266.5\end{array}$ & $\begin{array}{l}245.9 \\
256.9 \\
260.6 \\
264.2 \\
271.5\end{array}$ \\
\hline $\begin{array}{l}53.1 \\
52.7 \\
53.3 \\
53.5 \\
53.4\end{array}$ & $\begin{array}{l}25.0 \\
24.1 \\
22.4 \\
19.4 \\
18.3\end{array}$ & $\begin{array}{l}21.9 \\
23.2 \\
24.3 \\
27.1 \\
28.3\end{array}$ & $\begin{array}{l}257.1 \\
261.3 \\
265.0 \\
272.9 \\
276.9\end{array}$ & $\begin{array}{l}260.5 \\
264.2 \\
267.4 \\
275.6 \\
279.2\end{array}$ & $\begin{array}{l}263.9 \\
267.1 \\
270.3 \\
278.8 \\
282.2\end{array}$ & $\begin{array}{l}269.0 \\
272.4 \\
275.4 \\
282.8 \\
284.9\end{array}$ & $\begin{array}{l}274.6 \\
280.9 \\
279.5 \\
294.6 \\
289.1\end{array}$ \\
\hline $\begin{array}{l}53.6 \\
53.6 \\
53.7 \\
54.1 \\
54.7\end{array}$ & $\begin{array}{r}16.9 \\
15.5 \\
12.6 \\
8.2 \\
24.3\end{array}$ & $\begin{array}{l}29.5 \\
30.9 \\
33.7 \\
37.7 \\
21.0\end{array}$ & $\begin{array}{l}270.3 \\
280.9 \\
292.6 \\
313.0 \\
265.1\end{array}$ & $\begin{array}{l}273.0 \\
283.3 \\
\cdots\end{array}$ & $\begin{array}{l}275.7 \\
285.1 \\
-\end{array}$ & $\begin{array}{l}279.0 \\
287.1 \\
\cdots\end{array}$ & $\begin{array}{l}282.8 \\
297.3 \\
-\end{array}$ \\
\hline $\begin{array}{l}55.2 \\
56.1 \\
56.4 \\
56.9 \\
56.9\end{array}$ & $\begin{array}{l}25.9 \\
41.6 \\
38.7 \\
35.9 \\
33.2\end{array}$ & $\begin{array}{r}18.9 \\
2.3 \\
4.9 \\
7.2 \\
9.9\end{array}$ & $\begin{array}{l}258.3 \\
227.6 \\
232.4 \\
238.8 \\
241.5\end{array}$ & $\begin{array}{l}261.8 \\
231.4 \\
236.5 \\
243.2 \\
246.2\end{array}$ & $\begin{array}{l}265.7 \\
236.1 \\
241.9 \\
247.3 \\
251.1\end{array}$ & $\begin{array}{l}270.6 \\
242.1 \\
246.8 \\
252.0 \\
256.7\end{array}$ & $\begin{array}{l}276.0 \\
247.8 \\
253.5 \\
257.7 \\
264.3\end{array}$ \\
\hline $\begin{array}{l}57.3 \\
56.9 \\
57.1 \\
57.1 \\
57.3\end{array}$ & $\begin{array}{l}33.7 \\
30.8 \\
30.7 \\
28.1 \\
28.0\end{array}$ & $\begin{array}{r}9.0 \\
12.3 \\
12.2 \\
14.8 \\
14.7\end{array}$ & $\begin{array}{l}244.8 \\
248.3 \\
248.5 \\
254.2 \\
254.4\end{array}$ & $\begin{array}{l}249.6 \\
253.0 \\
252.6 \\
258.9 \\
259.3\end{array}$ & $\begin{array}{l}254.3 \\
258.3 \\
257.5 \\
263.4 \\
264.1\end{array}$ & $\begin{array}{l}259.6 \\
263.0 \\
261.8 \\
268.2 \\
269.0\end{array}$ & $\begin{array}{l}268.0 \\
269.0 \\
267.3 \\
275.1 \\
275.1\end{array}$ \\
\hline $\begin{array}{l}57.4 \\
57.5 \\
58.6 \\
57.5 \\
58.8\end{array}$ & $\begin{array}{l}25.3 \\
22.8 \\
19.9 \\
20.1 \\
17.4\end{array}$ & $\begin{array}{l}17.3 \\
19.7 \\
21.5 \\
22.4 \\
23.8\end{array}$ & $\begin{array}{l}261.7 \\
268.0 \\
277.1 \\
272.6 \\
287.8\end{array}$ & $\begin{array}{l}266.5 \\
271.5 \\
281.2 \\
277.2 \\
290.1\end{array}$ & $\begin{array}{l}270.3 \\
274.7 \\
284.9 \\
280.5 \\
-\end{array}$ & $\begin{array}{l}274.8 \\
279.5 \\
288.9 \\
285.5 \\
----\end{array}$ & $\begin{array}{l}282.0 \\
286.7 \\
297.3 \\
294.0 \\
\end{array}$ \\
\hline $\begin{array}{l}57.7 \\
58.5 \\
58.0 \\
58.5 \\
58.4\end{array}$ & $\begin{array}{r}17.4 \\
26.3 \\
12.2 \\
6.2 \\
35.7\end{array}$ & $\begin{array}{r}24.9 \\
15.2 \\
29.8 \\
35.3 \\
5.9\end{array}$ & $\begin{array}{l}277.1 \\
260.9 \\
297.8 \\
325.6 \\
242.7\end{array}$ & $\begin{array}{l}282.2 \\
265.7 \\
301.6\end{array}$ & $\begin{array}{l}285.4 \\
270.6 \\
304.7\end{array}$ & $\begin{array}{l}288.8 \\
276.0 \\
-258.8\end{array}$ & $\begin{array}{l}296.1 \\
283.4 \\
-\end{array}$ \\
\hline $\begin{array}{l}59.6 \\
60.4 \\
60.5 \\
60.7 \\
60.8\end{array}$ & $\begin{array}{l}37.5 \\
37.4 \\
34.9 \\
32.4 \\
29.8\end{array}$ & $\begin{array}{l}2.9 \\
2.2 \\
4.6 \\
6.9 \\
9.4\end{array}$ & $\begin{array}{l}238.3 \\
239.0 \\
244.0 \\
249.5 \\
254.2\end{array}$ & $\begin{array}{l}243.9 \\
246.6 \\
250.1 \\
255.3 \\
260.5\end{array}$ & $\begin{array}{l}249.6 \\
252.2 \\
255.9 \\
261.0 \\
266.8\end{array}$ & $\begin{array}{l}255.4 \\
257.6 \\
261.3 \\
267.1 \\
272.3\end{array}$ & $\begin{array}{l}268.3 \\
272.6 \\
277.8\end{array}$ \\
\hline $\begin{array}{l}61.1 \\
61.2 \\
61.5 \\
61.7 \\
61.5\end{array}$ & $\begin{array}{l}27.0 \\
24.4 \\
21.6 \\
19.2 \\
18.3\end{array}$ & $\begin{array}{l}11.9 \\
14.4 \\
16.9 \\
19.1 \\
20.2\end{array}$ & $\begin{array}{l}260.8 \\
265.7 \\
272.8 \\
279.5 \\
284.2\end{array}$ & $\begin{array}{l}266.5 \\
271.7 \\
278.7 \\
284.9 \\
289.4\end{array}$ & $\begin{array}{l}271.9 \\
277.5 \\
283.9 \\
289.5 \\
292.5\end{array}$ & $\begin{array}{l}277.5 \\
232.5 \\
287.4 \\
293.2 \\
297.5\end{array}$ & $\begin{array}{l}284.1 \\
290.0 \\
295.4 \\
304.0 \\
304.0\end{array}$ \\
\hline $\begin{array}{l}61.8 \\
62.0 \\
62.0 \\
62.4 \\
62.6\end{array}$ & $\begin{array}{r}16.7 \\
14.2 \\
11.6 \\
9.0 \\
5.8\end{array}$ & $\begin{array}{l}21.5 \\
23.8 \\
26.4 \\
28.6 \\
31.6\end{array}$ & $\begin{array}{l}287.6 \\
296.2 \\
306.6 \\
318.8 \\
335.5\end{array}$ & $\begin{array}{l}292.6 \\
300.9 \\
311.1 \\
322.1 \\
-\end{array}$ & $\begin{array}{l}296.9 \\
304.3 \\
313.5 \\
-\end{array}$ & \begin{tabular}{c}
299.5 \\
\hdashline.- \\
.-- \\
.--
\end{tabular} & \begin{tabular}{l}
$-\cdot-$ \\
$-\cdots$ \\
\hdashline$-\cdot$ \\
$-\cdots$
\end{tabular} \\
\hline $\begin{array}{l}64.1 \\
64.3 \\
64.9 \\
65.2 \\
64.9\end{array}$ & $\begin{array}{l}31.3 \\
28.9 \\
26.1 \\
21.1 \\
21.2\end{array}$ & $\begin{array}{r}4.6 \\
6.8 \\
9.0 \\
13.7 \\
13.9\end{array}$ & $\begin{array}{l}252.8 \\
256.2 \\
262.9 \\
275.7 \\
275.3\end{array}$ & $\begin{array}{l}259.3 \\
263.5 \\
269.9 \\
282.2 \\
282.1\end{array}$ & $\begin{array}{l}265.5 \\
269.8 \\
276.3 \\
288.1 \\
287.9\end{array}$ & $\begin{array}{l}271.7 \\
270.1 \\
281.6 \\
293.0 \\
293.0\end{array}$ & $\begin{array}{l}- \\
288.1 \\
299.4 \\
299.6\end{array}$ \\
\hline $\begin{array}{l}65.2 \\
65.2 \\
65.5 \\
65.6 \\
65.5\end{array}$ & $\begin{array}{l}18.8 \\
18.7 \\
16.1 \\
16.1 \\
13.7\end{array}$ & $\begin{array}{l}16.0 \\
16.1 \\
18.4 \\
18.3 \\
20.8\end{array}$ & $\begin{array}{l}280.6 \\
283.0 \\
289.0 \\
290.2 \\
299.9\end{array}$ & $\begin{array}{l}286.6 \\
288.7 \\
295.3 \\
296.1 \\
305.3\end{array}$ & $\begin{array}{l}292.6 \\
293.3 \\
300.3 \\
300.5 \\
309.0\end{array}$ & $\begin{array}{l}296.4 \\
297.9 \\
305.4 \\
305.6 \\
-\end{array}$ & \begin{tabular}{l}
307.9 \\
311.4 \\
\hdashline- \\
\hdashline
\end{tabular} \\
\hline $\begin{array}{l}65.8 \\
67.8 \\
68.0 \\
67.8 \\
69.8\end{array}$ & $\begin{array}{l}10.9 \\
19.9 \\
17.3 \\
15.4 \\
19.3\end{array}$ & $\begin{array}{l}23.3 \\
12.3 \\
14.7 \\
16.8 \\
10.9\end{array}$ & $\begin{array}{l}309.7 \\
280.2 \\
287.7 \\
295.8 \\
282.9\end{array}$ & $\begin{array}{l}314.3 \\
287.0 \\
294.2 \\
302.0 \\
290.1\end{array}$ & $\begin{array}{l}324.4 \\
293.0 \\
299.8 \\
307.6 \\
296.3\end{array}$ & $\begin{array}{l}298.2 \\
304.4 \\
311.9 \\
302.5\end{array}$ & \begin{tabular}{l}
302.7 \\
- \\
\hdashline-
\end{tabular} \\
\hline $\begin{array}{l}70.0 \\
70.6\end{array}$ & $\begin{array}{l}17.0 \\
14.1\end{array}$ & $\begin{array}{l}13.0 \\
15.3\end{array}$ & $\begin{array}{l}289.9 \\
298.3\end{array}$ & $\begin{array}{l}297.4 \\
305.8\end{array}$ & $\begin{array}{l}303.6 \\
311.6\end{array}$ & $\begin{array}{l}309.2 \\
316.9\end{array}$ & - \\
\hline
\end{tabular}

a Average composition of before and after analyses except for two-liquid compositions, which are batch compositions except as noted by footnote d.

sitions, which are batch compositions except as noted by footnote d.
b Interpolated values. The temperature of any measurement may have been b Interpolated values. T T
$+4^{\circ} \mathrm{C}$ from that given here.

$\pm 4^{\circ} \mathrm{C}$ from that given here.
c Glasses from binary alkaline-earth borate investigation [10].

d Average composition of two-liquid glasses made primarily for this investigation. Analyzed values before surface-tension measurement. Glasses not indicated by footnotes $\mathrm{c}$ or $\mathrm{d}$ were from an investigation of various properties of the system $\mathrm{BaO}-\mathrm{B}_{2} \mathrm{O}_{3}-\mathrm{SiO}_{2}$ by Hamilton, Cleek, and Grauer [17]. 


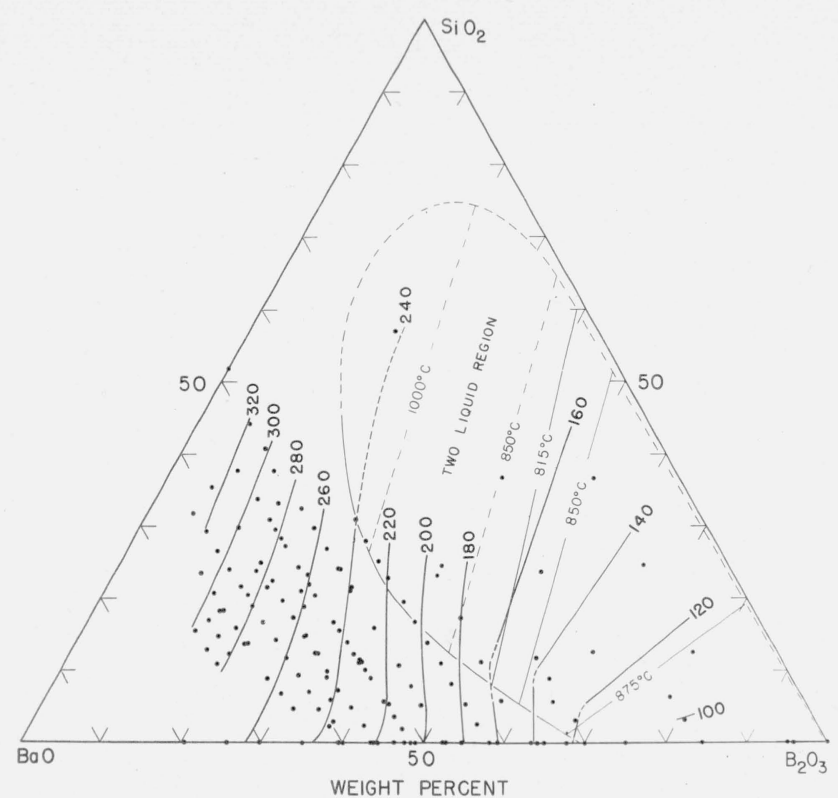

Figure 1. Isoepitatic (constant surface-tension) lines of the system $\mathrm{BaO}-\mathrm{B}_{2} \mathrm{O}_{3}-\mathrm{SiO}_{2}$ at $1,300^{\circ} \mathrm{C}$ and locations of compositions studied.

The outline of the two-liquid immiscibility region after Levin and Ugrinic [16].

fact that at any given temperature the upper layer of all compositions along a conode is identical. The lighter liquid will tend to rise to the surface, but if there were incomplete separation, there may be some of the lower liquid in the upper layer. The completeness of separation becomes greater as one departs from the two-liquid interface; and at the surface, where the surface tension is measured, the minimum amount of the lower layer will be present. The presence of lower liquid in the upper liquid is indicated by opacity. The glass that adhered to the ring was clear, indicating the probable absence of any of the lower liquid in the surface under test. Therefore, surface measurements may give a good indication of the direction of the conodes in the twoliquid region, and surface-tension measurements may offer another approach to the study of phase equilibria in the two-liquid region.

Figure 2 shows the isoepitatic lines at $1,100^{\circ} \mathrm{C}$. A comparison with the previous figure shows a slight counterclockwise rotation of the lines within the two-liquid region. This is in accord with the rotation of the conodes with temperacure reported in the phase work.

Figure 3 shows a plot of the isoepitatic lines at $900^{\circ} \mathrm{C}$. The changes in surface tension between $1,100^{\circ}$ and $900^{\circ} \mathrm{C}$ are similar to those between $1,300^{\circ}$ and $1,100^{\circ} \mathrm{C}$.

The constant surface-tension lines near the composition corresponding to the $3 \mathrm{BaO} \cdot 3 \mathrm{~B}_{2} \mathrm{O}_{3} \cdot 2 \mathrm{SiO}_{2}$ compound deviate from simple curves. This compound was characterized by Levin and Ugrinic [16] as one that melts congruently at $1,009^{\circ} \mathrm{C}$ and whose primary field occupies a large portion of the glass-forming region of the system. This is 109 $\operatorname{deg} \mathrm{C}$ above the temperature of the present measure-

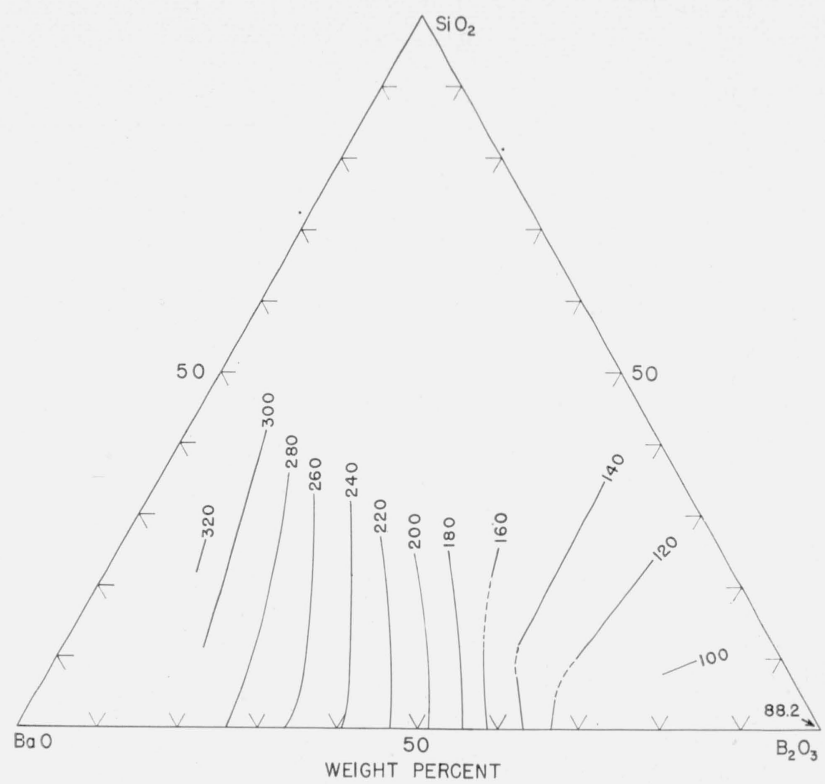

FIgure 2. Isoepitatic lines of the system $\mathrm{BaO}-\mathrm{B}_{2} \mathrm{O}_{3}-\mathrm{SiO}_{2}$ at $1,100^{\circ} \mathrm{C}$.

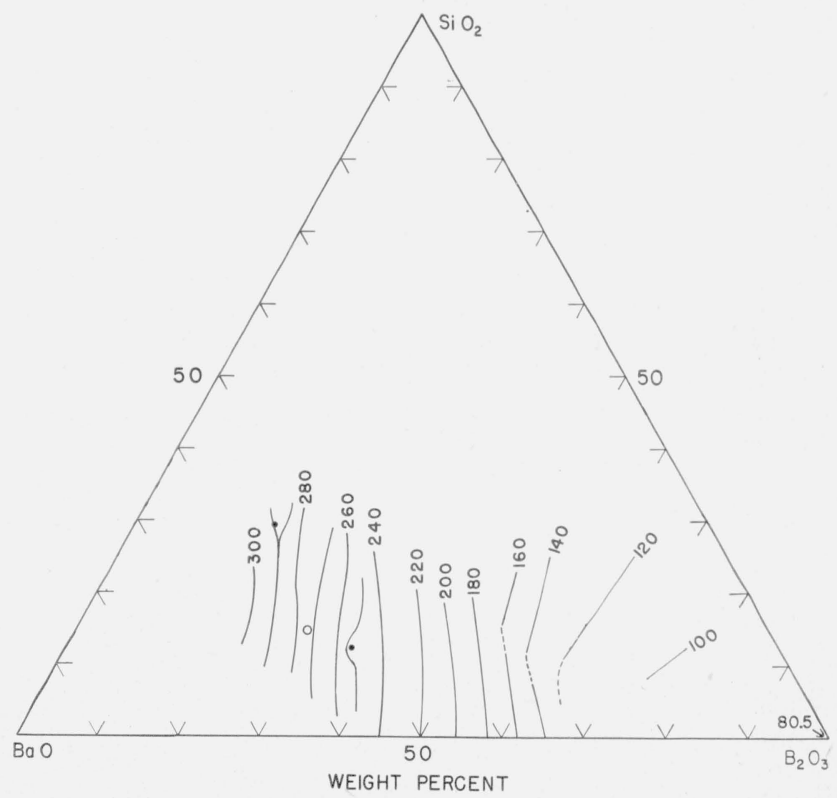

FIgure 3. Isoepitatic lines of the system $\mathrm{BaO}-\mathrm{B}_{2} \mathrm{O}_{3}-\mathrm{SiO}_{2}$ at $900^{\circ} \mathrm{C}$.

The open circle indicates the location of the $3 \mathrm{BaO} \cdot 3 \mathrm{~B}_{2} \mathrm{O}_{3} \cdot 2 \mathrm{SiO}_{2}$ compound, identified by Levin and Ugrinic [16]. The two closed circles indicate the locations of the low-surface-tension areas.

ment. The deviation of the isoepitatic lines is associated with an irregular surface-tension region near the compound (indicated by the open circle) and two relatively low surface-tension areas (closed circles).

Above the liquidus temperature $\left(1,009^{\circ} \mathrm{C}\right)$ at the compound composition no departures of the isoepitatic lines were observed. The possibility of the formation of crystals may be the cause of these 


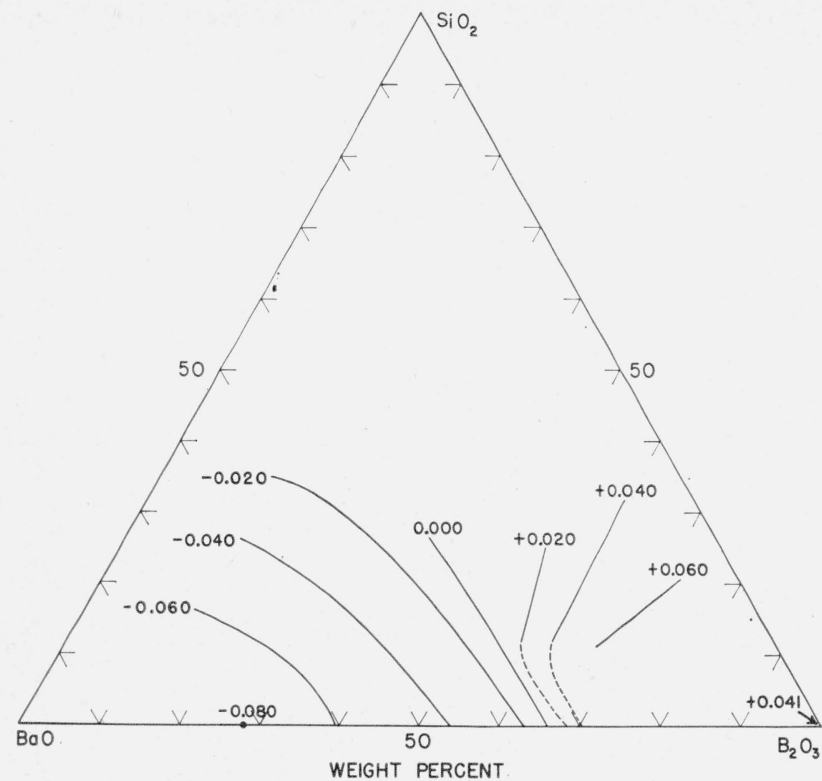

FIguRE 4. Constant coefficient of surface-tension lines of the system $\mathrm{BaO}-\mathrm{B}_{2} \mathrm{O}_{3}-\mathrm{SiO}_{2}$ across the range $1,000^{\circ}$ to $1,300^{\circ} \mathrm{C}$.

deviations from simple curves. Microscopic examination of the glasses has shown no sign of crystallization, and the region about the compound is known to produce quite stable glasses, i. e., the rate of crystal growth in this region is very low. No explanation is available for the minima.

Figure 4 shows the lines of constant-temperature coefficient of surface tension over the range $1,000^{\circ}$ to $1,300^{\circ} \mathrm{C}$. With decreasing temperature there is a decrease in surface tension within the two-liquid region and an increase over most of the area exterior to this region. The scatter of the points and the small range of the coefficients made it difficult to determine the exact position of the lines. For example, points with the value $-0.040($ dyne $/ \mathrm{cm}) /{ }^{\circ} \mathrm{C}$ are found in about three-fourths of the area between the -0.020 and $-0.060($ dyne $/ \mathrm{cm}) /{ }^{\circ} \mathrm{C}$ lines. The lines shown are correspondingly uncertain. The twoliquid region is characterized by the similarity between the constant coefficient lines and the conodes in the phase diagram. There is a maximum coefficient in this region, and a zero coefficient near the boundary. The values indicated in this figure are the average over the temperature range.

The surface-tension versus temperature curve for any particular composition is not a straight line and may contain inflections. In the region around the 0.000 (dyne $/ \mathrm{cm}) /^{\circ} \mathrm{C}$ line the curves may show maxima, minima, or both. It may also be seen from this figure that a change in composition in a given direction does not always cause a similar change in the coefficient of surface tension, and as noted earlier, it does not always cause a similar change in surface tension.

\section{Summary and Conclusions}

The surface tension of a series of $\mathrm{BaO}-\mathrm{B}_{2} \mathrm{O}_{3}-\mathrm{SiO}_{2}$ compositions was measured over the range $900^{\circ}$ to $1,300^{\circ} \mathrm{C}$. In the two-liquid region the values of surface tension and the coefficients of surface tension followed the conodes in the phase diagram., indicating a possible new technique for phase study in this type of region. In general, the surface tension increased with decrease in $\mathrm{B}_{2} \mathrm{O}_{3}$ content. At $900^{\circ} \mathrm{C}$ the lines of constant surface tension reflected an irregularity near the composition corresponding to the $3 \mathrm{BaO}$. $3 \mathrm{~B}_{2} \mathrm{O}_{3} \cdot 2 \mathrm{SiO}_{2}$ compound, which has a melting point of $1,009^{\circ} \mathrm{C}$.

\section{References}

[1] Leo Shartsis, Sam Spinner, and Alden W. Smock, Surface tensions of compositions in the systems $\mathrm{PbO}-\mathrm{B}_{2} \mathrm{O}_{3}$ and $\mathrm{PbO}-\mathrm{SiO}_{2}$, J. Am. Ceram. Soc. 31, 23 (1948); J. Research NBS 40, 61 (1948) RP1855.

[2] Leo Shartsis and Edwin S. Newman, Some energy relationships in the systems $\mathrm{PbO}-\mathrm{B}_{2} \mathrm{O}_{3}$ and $\mathrm{PbO}-\mathrm{SiO}_{2}$, J. Am. Ceram. Soc. 31, 213 (1948); J. Research NBS 40, 471 (1948) RP1893.

[3] Leo Shartsis and Rodrigo Canga, Surface tension of molten zinc borates, J. Research NBS 43, 221 (1949) RP2023; Ceram. Abstr. p. 110i. (1950).

[4] Leo Shartsis and Sam Spinner, Surface tension of molten alkali silicates, J. Research NBS 46, 385 (1951) RP2209.

[5] Leo Shartsis, Sam Spinner, and Webster Capps, Density, expansivity, and viscosity of molten alkali silicates. J. Am. Ceram. Soc. 35, 155 (1952).

[6] Leo Shartsis and Webster Capps, Surface tension of molten alkali borates, J. Am. Ceram. Soc. 35, 169 (1952).

[7] Leo Shartsis, Webster Capps, and Sam Spinner, Density and expansivity of alkali borates and density characteristics of some other binary glasses, J. Am. Ceram. Soc. 36, 35 (1953).

[8] L. Shartsis, W. Capps, and S. Spinner, Viscosity and electrical resistivity of molten alkali borates, J. Am. Ceram. Soc. 36, 319 (1953).

[9] L. Shartsis, and W. Capps, Energy relations in binary alkali borates, J. Am. Ceram. Soc. 37, 27 (1954).

[10] Leo Shartsis and H. F. Shermer, Surface tension, density, viscosity, and electrical resistivity of molten binary alkaline-earth borates, J. Am. Ceram. Soc. 3\%, 544 (1954).

[11] C. E. Weir and L. Shartsis, Compressibility of binary alkali borate and silicate glasses at high pressures, $\mathbf{J}$. Am. Ceram. Soc. 38, 299 (1955).

[12] C. E. Weir and L. Shartsis, Compressibility of binary alkaline-earth borate glasses at high pressures (unpublished manuscript).

[13] Herman F. Shermer, Thermal expansion of binary alkaline-earth borate glasses, J. Research NBS 56, 73 (1956) RP2650.

[14] Leo Shartsis and A. W. Smock, Surface tensions of some optical glasses, J. Am. Ceram. Soc. 30, 130 (1947).

[15] Edward W. Washburn, George Reed Shelton, and Earl E. Libman, The viscosities and surface tensions of the soda-lime-silica glasses at high temperatures (University of Illinois, Engineering Experiment Station Bulletin 140, 1924).

[16] Ernest M. Levin and George M. Ugrinic, The system barium oxide-boric oxide-silica, J. Reserach NBS 51, 37 (1953) RP2430.

[17] Edgar H. Hamilton, G. W. Cleek, and O. H. Grauer, Some properties of $\mathrm{BaO}-\mathrm{B}_{2} \mathrm{O}_{3}-\mathrm{SiO}_{2}$ glasses and a theory of glass structure (unpublished manuscript).

Washington, December 2, 1955. 